Published in final edited form as:

Methods Mol Biol. 2020 ; 2150: 25-44. doi:10.1007/7651_2019_232.

\title{
Long-Term Cell Fate Tracking of Individual Renal Cells Using Serial Intravital Microscopy
}

\author{
Ina Maria SchiessI ${ }^{1}$, Katharina Fremter ${ }^{2}$, James L. Burford ${ }^{3}$, Hayo Castrop ${ }^{2}$, Janos Peti- \\ Peterdi ${ }^{1}$ \\ ${ }^{1}$ Department of Physiology and Neuroscience, Zilkha Neurogenetic Institute, Keck School of \\ Medicine, University of Southern California, Los Angeles, USA \\ ${ }^{2}$ Institute of Physiology, University of Regensburg, Regensburg, Germany \\ ${ }^{3}$ Department of Ophthalmology, University of Southern California, Los Angeles, USA
}

\section{Abstract}

Intravital multiphoton microscopy of the kidney is a powerful technique to study alterations in tissue morphology and function simultaneously in the living animal and represents a dynamic and developing research tool in the field. Recent technological advances include serial intravital multiphoton microscopy of the same kidney regions over several weeks and combined with ex vivo histology for cellular biomarker expression of the same cells, which had been subject to serial imaging before. Thus, serial intravital multiphoton microscopy followed by ex vivo histology provides unique tools to perform long-term cell fate tracing of the same renal cells during physiological and pathophysiological conditions, thereby allowing the detection of structural changes of the same renal cells over time. Examples include renal cell migration and proliferation while linking these events to local functional alterations and eventually to the expression of distinct cellular biomarkers. Here, we provide a detailed step-by-step protocol to facilitate serial intravital multiphoton microscopy for long-term in vivo tracking of renal cells and subsequent ex vivo histology for immunohistological staining of the same cells in the fixed tissue.

\section{Keywords}

Intravital imaging; Multiphoton microscopy; Cell fate tracking; Fluorescent reporter; Tissue remodeling; Immunohistology

\section{Introduction}

In order to study renal structure and function in vivo and in situ, gold standard techniques have been available that include immunohistology, electron microscopy, micropuncture studies, and protocols to assess parameters of global kidney function, such as total body glomerular nitration rate (GFR), renal blood flow (RBF), and renal concentrating ability. Even though these approaches have proven very useful to study renal physiology and pathophysiology, they are limited to assess either structural or functional information, but not both together. Consequently, it is rather difficult to gain new insights on dynamic renal cell-to-cell communication and its functional consequences in a physiological and pathophysiological context. Intravital multiphoton microscopy (MPM) uniquely allows the 
investigator to simultaneously study renal structure and function at a cellular to subcellular resolution. First established nearly two decades ago, this approach provided important novel insights into renal physiology and pathophysiology [1, 2]. In contrast to conventional single photon microscopy, the required energy for fluorescent dye excitation is provided by two photons of longer wavelength, which reach the focal point simultaneously and hence are absorbed together. Since this physical event requires very high photon densities at the focal point, special pulsed lasers are used. This approach is very suitable for in vivo deep tissue imaging, because light of longer wavelength and lower energy involves less phototoxicityrelated tissue damage and less scattering for deeper tissue penetration $[2,3]$.

Chronic kidney disease is associated with heterogeneous histologic injury patterns, which contribute to progressive decline in renal function [4]. However, the underlying mechanisms for this structural and functional heterogeneity of individual nephrons are incompletely understood. Studying this phenomenon is difficult due to the lack of techniques, which allow the follow-up on individual nephrons and the generation of paired functional and morphological data over time. Recently established serial intravital MPM of the kidney first allowed us to overcome these limitations [5-7]. Thus, totally noninvasive and high-quality imaging of the kidney can be achieved via an abdominal imaging window (AIW) that consists of a coverslipped titanium ring, which is implanted into the mouse abdomen and to which the kidney is glued to [8]. This allows the investigator to navigate within a large kidney area and to reidentify and reimage the same kidney regions over several days or weeks. Hence, the generation of structural and functional data of individual nephrons over time makes it possible to (1) overcome renal tissue heterogeneity issues, (2) establish the dynamics and pattern of individual cells' motility and migration over time, and (3) correlate renal cell plasticity and cell-to-cell communication with functional alterations in vivo.

While serial intravital MPM of the kidney enables the simultaneous study of structural and functional changes over time, the in vivo staining of cellular biomarkers is hampered by the limited bioavailability of injected antibodies or dyes at the target cell. Consequently, probing for cellular biomarkers is typically done on histological sections and hence lacks any dynamic and functional information on the investigated cells. However, a recent study managed to reidentify the same kidney regions ex vivo, which had been subject to serial intravital imaging before, and to thereby link the expression of in vivo inaccessible cellular biomarkers to dynamic and functional information of the same cells [6].

The procedure for the implantation of abdominal imaging windows for liver, spleen, and kidney imaging was described recently [8]. Here, we provide an adapted protocol from Ritsma et al., specifically to facilitate renal cell fate tracking in the mouse kidney using serial intravital microscopy. We offer a comprehensive manual on how the same kidney regions can be reidentified over several days and weeks and how this can be combined with functional analysis using genetic reporter models or established injectable fluorescent dyes. Furthermore, we describe a novel protocol and show how the implantation of an abdominal imaging window can be used to reidentify the same kidney areas ex vivo for subsequent immunohistological staining of cellular biomarkers of interest. With the help of this approach, one can further push the boundaries of intravital imaging toward the assessment of structural, functional, and transcriptional characteristics of renal cells over time. 


\section{Materials}

\subsection{Material for AIW Preparation}

1. Custom-made abdominal imaging windows from titanium: consisting of an upper and a lower ring, separated by a $1.5 \mathrm{~mm}$ width groove (Fig. 1a):

a. Size 1 (mice $\geq 19 \mathrm{~g}$ body weight): $14 \mathrm{~mm}$ outer diameter, $8 \mathrm{~mm}$ inner diameter.

b. Size 2 (mice 13-18 g body weight): $12 \mathrm{~mm}$ outer diameter, $6 \mathrm{~mm}$ inner diameter.

2. 12-mm-diameter coverslips (Carolina Assistant-Brand).

3. $70 \%$ ethanol (Sigma).

4. $100 \%$ acetone (Sigma).

5. Sterile $0.9 \%$ saline (Sigma).

6. Instant glue: Sekunden Kleber (Uhu).

7. Instant glass glue: Loctite glass glue (Henkel).

8. Poly(L-amino acid)-polyethylene glycol/poly(L-lysine)-polyethylene glycol (PLL-g-PEG, Nanosoft Polymers).

9. Prepare sterile $1 \mathrm{ng} / \mathrm{ml}$ PLL-g-PEG solution in $10 \mathrm{mM}$ HEPES buffer, $\mathrm{pH}$ 4.7.

\subsection{Surgical Instruments and Equipment}

1. Extra fine Graefe forceps (Fine Science Tools).

2. Straight Bonn scissors (Fine Science Tools).

3. Mayo-Hegar needle holders (Fine Science Tools).

4. Sterile cotton-tipped applicators (Puritan).

5. Nonabsorbable monofilament surgical suture: 6-0 nylon (AD Surgical).

6. Buprenorphine slow release formulation: Buprenorphine SR-LAB $0.5 \mathrm{mg} / \mathrm{ml}$ (Zoopharm).

7. Sterile towel drapes (Dynarex).

8. Isoflurane vaporizer: SomnoSuite Small Animal Anesthesia System (Kent Scientific).

9. Heated operating table: SurgiSuite (Kent Scientific).

10. Surgery lighting: KL1500LCD (Zeiss).

\subsection{Serial Intravital Imaging}

1. Leica SP8 DIVE MP system:

a. DMi8CEL advanced modular inverted microscope. 
b. $\quad 680-1300 \mathrm{~nm}$ extended IR spectrum Discovery MP laser from Coherent.

c. Ultrahigh-speed resonant scanner $(8-12 \mathrm{kHz})$.

d. Quad module four spectral HyD SP GaAsP detectors (from Leica Microsystems).

e. $40 \times / 1.10 \mathrm{NA}$ water objective.

2. Custom-made microscope insert equipped with a coverslip.

3. Isoflurane vaporizer: SomnoSuite Small Animal Anesthesia System (Kent Scientific).

4. Noninvasive tail-cuff blood pressure monitoring including heating pad: CODA monitor (Kent Scientific).

5. Artificial Tears Ointment (Rugby).

6. Syringes for fluorescent dye application: BD Insulin Syringes Ultra-Fine needles 30G (Beckton Dickson).

7. Fluorescent dyes: see Table 1.

\subsection{Ex Vivo Histology Preparation}

1. $10 \%$ neutral formalin solution (Sigma).

2. Sterile PBS (Sigma).

3. $70 \%$ ethanol (Sigma).

4. Bovine serum (Sigma).

5. Horse Serum (Sigma).

6. $\quad 0.5 \mathrm{~mm}$ and $1 \mathrm{~mm}$ spacers (SunJin Lab).

7. VWR micro cover glass No. $124 \times 40 \mathrm{~mm}$ (VWR).

\section{Methods}

\subsection{Abdominal Imaging Window (AIW) Preparation}

1. Apply a thin layer of cyanoacrylate-based glass glue on the upper titanium ring of the AIW, so that all the surface that will be in contact with the coverslip is covered. Regular cyanoacrylate glue may also be used, but the results are less accurate.

2. Place a $12 \mathrm{~mm}$ coverslip on the glass glue-covered titanium ring using forceps, and apply gentle pressure for 1 min using a cotton swab.

3. Let the glue dry for $1 \mathrm{~h}$.

4. If necessary, remove excessive glue from the AIW using a cotton swab soaked into $100 \%$ acetone. 
5. Test for water tightness by placing the dry AIW upside down onto a paper tissue and fill it with saline. There should be no leak of water onto the paper tissue (see Note 1).

6. Sterilize the coverslipped AIW by incubating it in $70 \%$ (vol/vol) ethanol for 30 min.

7. Optional PLL-g-PEG solution coverage: Place the window upside down into a sterile flow cabinet, and fill its interior side with $150 \mu \mathrm{l}$ of $1 \mathrm{ng} / \mathrm{ml}$ PLL-g-PEG solution. Let the solution incubate for $1 \mathrm{~h}$, and then remove the solution, and wash the window with PBS (see Note 2).

\subsection{Surgery Preparation}

1. Sterilize surgical tools by autoclaving or by incubation in a dry sterilizer for 10 $\min$.

2. Disinfect surgical equipment (bench, operating table, etc.) with $70 \%$ ethanol.

3. Induce anesthesia by placing the mouse into an induction chamber flooded with $2.5 \%$ isoflurane.

4. Once the mouse is asleep, use an electrical shaver to shave the left flank, and place it on its side onto an operating table with a servo-controlled heating plate. Use $1.5 \%$ isoflurane delivered by a nose mask to maintain the anesthesia.

5. Apply eye ointment onto the eyes of the mouse.

6. Apply $1 \mathrm{mg} / \mathrm{kg}$ of buprenorphine slow release formulation s.c. into the neck fold of the mouse.

7. Disinfect the shaved skin of the flank using $70 \%$ ethanol and betadine or chlorhexidine using a circular application approach three times starting from inside and rotating outward.

8. Cover the mouse with a sterile surgical drape, which is equipped with a $1.5 \mathrm{~cm}$ opening in diameter, and place the opening above the left flank (Fig. 1b).

\subsection{Surgical AIW Implantation}

1. Make a 1-cm-long dorsoventral incision above the left kidney.

2. Perform a purse-string suture to attach the skin to the muscle layer of the abdominal wall, using nonabsorbable 0.7 monofilament nylon surgical suture (Fig. 1b, see Note 3).

3. Use a 10 il pipette tip to precisely apply cyanoacrylate glue onto the dry interior ring surface of the AIW, where the coverslip is attached to the titanium ring.

4. Place the AIW carefully onto the kidney surface using forceps, and hold it in place for 5 min until the glue is dry while applying gentle pressure onto the organ (see Note 4). 
5. Use forceps to gently place the purse-string-sutured abdominal wall and skin into the groove of the window. Be careful not to detach the kidney from the coverslip (Fig. 1c).

6. Gently pull the loops of the purse-string to tighten the abdominal wall and skin around the AIW. Then, carefully place a double knot to fix the purse-string suture. Make sure the knots are hidden in the groove of the window, so that the mouse cannot access the suture and bite it open (Fig. 1d, e).

7. For recovering, relocate the mouse in a separate cage onto a heating pad for 30 min. Then place it back into its housing cage.

\subsection{Serial Intravital Imaging}

1. Induce anesthesia by placing the mouse into an induction chamber flooded with $2.5 \%$ isoflurane.

2. Optional: once the mouse is properly anesthetized, apply fluorescent dyes by retro-orbital injection or by tail vein injection. See Table 1 for an overview of suitable fluorescent dyes for in vivo labeling.

3. Relocate the mouse onto the microscope insert. The microscope insert can either have a customized hole to exactly fit the window [8] or be equipped with a coverslip. Note: the kidney can be imaged through two coverslips (one coverslip of the microscope insert, one coverslip of the AIW itself) without any noticeable optical limitations.

4. Place the nose of the mouse into a mask providing $1.5 \%$ isoflurane to maintain anesthesia, and place a heating pad onto the animal to maintain its body temperature at $37^{\circ} \mathrm{C}$.

5. Optional: Blood pressure can be monitored noninvasively via tail-cuff measurements (CODA monitor system, Kent Scientific, see Note 5).

6. For serial imaging, the animal must be placed in the same orientation for each individual imaging session. To achieve this, a customized imaging chamber which places the window in the same reproducible position and angle is helpful [8] but not necessary [6]. To find the exact same cortical kidney region in consecutive imaging sessions over several days, one or combinations of the following options can be used:

a. Morphological clues: The kidney is a structurally heterogeneous organ. The existence of superficial glomeruli, patterns of convoluting (proximal tubules), or branching tubules (connecting tubules) can be unique and useful to reidentify the exact same cortical regions over several weeks.

b. Reidentification of the same kidney regions can be simplified when using fluorescent reporter mouse models. Heterogeneous labeling of single cells in the kidney provides colorful patterns that can be easily recognized and reidentified (Fig. 2a). 
c. Motorized stage: Overview scans provide a map of the kidney surface attached to the coverslip (Fig. 2a). The same individual imaging areas can be found based on morphological clues or by storing and reloading of the coordinates of individual regions.

d. Non-motorized stage: If the equipment doesn't allow to create overview images or tile scans, the same cortical regions can also be found by recognizing morphological clues, such as glomeruli, etc., through the eyepiece or by manually scanning of the available kidney surface. Alternatively, an objective of lower magnification can be used to generate overview images of the kidney tissue attached to the coverslip.

e. Creating landmarks using laser injury. In order to mark and then find the same areas, a small local injury can be created by focusing high laser power on certain kidney structures, such as a glomerulus. This creates a characteristic highly autofluorescent spot, which can be found for several days (Fig. 2d, see Note 6).

\subsection{Serial Intravital Imaging}

1. Cell fate tracing using the Brainbow Confetti reporter model: Homozygous Confetti reporter mice allow the Cre-mediated cell-type-specific expression of two out of four possible fluorescent proteins per cell - membranous CFP, nuclear GFP, cytosolic YFP, and cytosolic RFP [24]. This results in a unique ID tag of individual cells by one out often potential color combinations [25]. In addition to that, the renal intravascular space can be visualized using a far-red fluorescent dye, conjugated to albumin (Fig. 2a, b) or to a high molecular weight dextran. Migration and proliferation of individually fluorescent-tagged cells can be detected and analyzed from 3D optical sectioning data derived from the same kidney regions at different time points (Fig. 3).

Using the novel Leica SP8 MP DIVE system, the four Confetti colors, Alexa 680-conjugated albumin, and second harmonic generation (SHG) signal can be excited (generated) and sufficiently separated in only two sequential tracks:

a. Track one: Excitation, $950 \mathrm{~nm}$; emission, 500-510 nm for GFP, 520$545 \mathrm{~nm}$ for YFP, 600-630 $\mathrm{nm}$ for RFP, 720-750 nm for Alexa 680ablumin

b. Track two: Excitation, $860 \mathrm{~nm}$; emission, 425-435 nm for SHG, 460 $485 \mathrm{~nm}$ for CFP

2. Complementing cell fate tracking with functional measurements of individual nephrons: Serial intravital MPM of the kidney provides cellular and subcellular resolution of structural changes over several days and weeks. In addition, injection of fluorescent dyes allows the measurement of alterations in renal function, such as of renal capillary blood flow [15, 22, 26], single-nephron glomerular filtration rate [22, 23], detection of intracellular redox state, mitochondrial function $[16,17]$, reactive oxygen species [15], protein 
endocytosis [6, 9], and lysosomal degradation [9]. For practical descriptions, please refer to detailed protocols published elsewhere [14, 22, 27]. Table 1 provides an overview of endogenously expressed autofluorescent molecules and established injectable fluorescent dyes to determine renal function in vivo.

3. Imaging of intracellular calcium levels using the GCaMP5 reporter model: This reporter model enables the Cre-mediated cell type-specific expression of the calcium-sensitive fluorescence indicator GCaMP5G and the calcium-insensitive fluorescent protein tdTomato [28]. GCaMP5G is expressed cytosolically, and its fluorescent intensity depends on realtime intracellular calcium levels in the cell. tdTomato is expressed in the cytosol and in the nuclei of recombinant cells and hence allows to distinguish between individual cells. Intracellular calcium levels can be assessed in a semi-ratiometric fashion, by normalizing GCaMP5G fluorescence over the calcium-insensitive fluorescence of tdTomato.

a. GCaMP5G and tdTomato are best excited at $940 \mathrm{~nm}$ (2-photon excitation maximum of GFP). Collect GCaMP5G and tdTomato emission at $490-530 \mathrm{~nm}$ and $570-630 \mathrm{~nm}$, respectively.

b. Keep the gain for the detection of GCaMP5G and tdTomato fluorescence consistent throughout all experiments. For semiquantitative assessment of intracellular calcium levels, determine GCaMP5G/tdTomato fluorescence ratio at comparable tissue depth (green fluorescence shows higher scattering in deep tissue regions), and excite with comparable laser power (see Note 7).

c. Plasma can be simultaneously visualized after injection of Alexa 680albumin. Collect Alexa 680-albumin emission at 680-750 nm.

4. Use of the 2-photon laser as a micromanipulator: Focused high laser power can be used to stimulate individual renal cells or to introduce hemodynamic irregularities (e.g., laser-induced thrombus formation). Hence, the MPM laser can be used as a micromanipulator using a three-step protocol (Fig. 4):

a. Step 1: Record baseline calcium fluctuations (using a GCaMP5 reporter model)/renal hemodynamics (after injection of fluorescent albuminconjugated dye) by performing a time-lapse (xyt) recording of a region of interest in the mouse kidney.

b. Step 2: Stimulate an individual cell by focusing the laser beam on a $1 \times$ 1 im square-shaped region of interest for $2 \mathrm{~s}$ using $100 \%$ laser power $(940 \mathrm{~nm}, 1.65 \mathrm{~W})$ at a depth of $60 \mu \mathrm{m}$ underneath the kidney capsule. Note: the settings for laser-induced cell stimulation are system-specific and depend in general on the laser output power, the used wavelength, the laser exposure time, the tissue depth, and the presence of fluorescent proteins or dyes in the target cell. For reproducible effects of laserinduced cell stimulation, suitable settings with respect to these factors need to be established beforehand. 
c. Step 3: Zoom out and perform a several minute-long time-lapse recording of the subsequent functional alterations. Apply the same imaging settings which were used for baseline recording. This step is time-sensitive and needs to be performed as quickly as possible after laser-induced cell stimulation ( see Note 8).

d. A combined three-step protocol can be applied by manually setting up each of the above three steps or by applying a fluorescence recovery after photobleaching (FRAP)-software plugin, which allows the presetting and recording of all three steps in one recording and file (faster, recommended).

\subsection{In Vivo Imaging Followed by Ex Vivo Histology of the Same Cortical Kidney Regions}

1. In order to find the same cortical kidney regions ex vivo, the use of laser-induced landmarks is recommended. At the end of the last imaging session (preferably immediately preceding tissue fixation), use focused high laser power for generating distinct local tissue damage and several visible, autofluorescent laser marks surrounding the regions of interest. Focus the laser beam on a $5 \times 5$ im square-shaped region, e.g., a spare glomerulus, for $5 \mathrm{~s}$ using $100 \%$ laser power $(940 \mathrm{~nm}, 1.65 \mathrm{~W})$ at a depth of $60 \mu \mathrm{m}$ underneath the kidney capsule. In order to avoid tissue damage of the kidney regions of interest, make sure that the laserinduced landmarks are placed in sufficient distance (Fig. 2d).

2. Anesthetize the animal by injecting ketamine/xylazine $(100 / 10 \mathrm{mg} / \mathrm{kg}$ body weight) i.p., and place in a flow cabinet.

3. Confirm that the animal is fully anesthetized by pinching its tail with tweezers. Then perform a large longitudinal incision to expose the heart and the abdominal vena cava.

4. Perfuse the animal with $10 \mathrm{ml}$ ice-cold PBS followed by $10 \mathrm{ml}$ of $10 \%$ formalin by infusing the fluids with constant pressure through the heart. Drain the blood and fluids through an incision of the abdominal vena cava, caudal of the kidneys.

5. Carefully remove the kidney from AIW, and postfix the tissue in $10 \%$ formalin for $20 \mathrm{~min}$.

6. Pause point: Optional, the kidney tissue can be stored in PBS overnight at $4{ }^{\circ} \mathrm{C}$.

7. The kidney area, which was attached to the coverslip, can be identified macroscopically by a characteristic bulge of the tissue surface. Separate the tissue of interest as a 1-2 mm thick tissue slice using a sharp razor blade (Fig. 1f).

8. Carefully remove the kidney capsule of the recovered tissue under a dissecting microscope using forceps.

9. Wash the tissue slice twice in PBS for $15 \mathrm{~min}$ at room temperature.

10. Block the tissue in $1 \% \mathrm{BSA} / 10 \%$ horse serum/PBS for $1 \mathrm{~h}$ at room temperature. 
11. Incubate the tissue in primary antibody ( $1: 100$ in $1 \% \mathrm{BSA} / 10 \%$ horse serum/ PBS) overnight at $4{ }^{\circ} \mathrm{C}$.

12. Wash the tissue twice with $1 \times \mathrm{PBS}$ at room temperature.

13. Incubate the tissue with the secondary antibody (1:400 in 1\% BSA/10\% horse serum/PBS) for $4 \mathrm{~h}$ at room temperature and protect from light.

14. Endogenous fluorescence stays preserved. Hence, secondary antibodies must be chosen in a way that the fluorescent staining can be spectrally and/or morphologically distinguished from endogenous fluorescence in the tissue (nuclear expression of green GFP vs. membranous binding of green Alexa 488anti-villin antibody, Fig. 3).

15. Wash the tissue twice with PBS.

16. Embed the tissue slice in PBS between two glass coverslips using 1-2 mm thick plastic spacers (Fig. 1j). Be sure that the kidney tissue, which was attached to the coverslip of the AIW, is now attached to the coverslip of the ex vivo preparation. Protect from light!

17. Place the ex vivo preparation onto the same microscope that was used for in vivo imaging, thereby ensuring that the kidney tissue is oriented in the same way as for previous in vivo imaging.

18. In order to find the same tissue regions as imaged previously in vivo, the same strategies may be applied as suggested for serial intravital microscopy (see Subheading 3.4, step 6). The strong autofluorescence of laser-induced landmarks can be readily detected on overview scans or when manually scanning the kidney surface touching the coverslip and help to reidentify the same kidney regions, previously imaged in vivo.

19. Once identified, reimage the regions of interest of the fixed tissue after adjusting the laser and detector settings with respect to the fluorescent-conjugated secondary antibodies used for the staining.

\subsection{Reusing of the AIW}

1. Thoroughly clean the AIW from skin and blood using a small brush, soap, and hot water.

2. Incubate the AIW in $100 \%$ acetone overnight to remove the coverslip and glue residues on the titanium ring.

3. $\quad$ Proceed with Subheading 2.1, item 1.

\subsection{Application of Serial Intravital MPM of the Mouse Kidney}

Serial intravital MPM is a powerful novel advancement of in vivo imaging of the kidney, which allowed us for the first time to extend the duration of imaging from only a few hours at a time to several days and weeks. Thus, the repeated optical sectioning of the same kidney structures in combination with state-of-the-art genetic reporter models allows investigators 
to follow cell-level renal tissue remodeling, individual cell migration, and proliferation with high spatial and temporal resolution and to overcome tissue heterogeneity issues. Consistently, the first study to perform serial intravital MPM of the kidney provided important clues against the traditional static view and found a rather dynamic nature of glomerular podocytes. Consecutive imaging of the same glomeruli during the development of several disease processes and models was successful in tracking the fate of individually labeled podocytes, by podocyte-specific expression of CFP, GFP, YFP, or RFP. Hence, the migration and clustering of podocytes during disease were documented based on paired observations and associated with the formation of thin nanotubules between the glomerular visceral and parietal cell layers [5].

Importantly, the tracking of individual cells over time can be combined with functional analysis, such as measurements of local glomerular permeability $[29,30]$, changes in glomerular and peritubular hemodynamics $[9,26]$, the homing of and the interaction with immune cells [17], as well as the assessment of intracellular calcium levels using cellspecific calcium-sensitive genetic reporter models [6, 12]. Recently, the cell-specific genetic expression of the fluorescent calcium indicator GCaMP3 allowed the assessment of intracellular calcium levels in podocytes with respect to structural and functional alterations. Increased podocyte calcium was suggested to dysregulate functional podocyte integrity by foot process actin remodeling [31] leading to slit diaphragm disruption and foot process effacement [32]. Intravital imaging of GCaMP3-expressing podocytes during renal fibrosis and glomerulosclerosis enabled the detection of locally increased albumin permeability of clustered and damaged podocytes and first visualized these phenomena simultaneously with increased podocyte calcium levels [12].

Furthermore, the 2-photon laser can be used as a micromanipulator to specifically target and manipulate single cells in vivo. Thus, the laser-induced stimulation of a single podocyte triggered the cell-to-cell propagation of a podocyte calcium wave across the affected glomerulus, which was associated with increased albumin permeability. Interestingly, cellto-cell podocyte calcium propagation and changes in albumin permeability were reduced in the presence of the nonselective purinergic receptor antagonist suramin. This suggested that podocytes are functionally coupled and communicate with each other via extracellular ATP release and signaling, which may also regulate glomerular albumin permeability [12]. Laserinduced cell stimulation can also be used to induce disruptions of the capillary wall to establish single-nephron hematuria [1] or thrombus formation [33]. Accordingly, here we provide a detailed protocol how the 2-photon laser can be used as micromanipulator (E.4) to analyze cell-to-cell calcium propagations in response to single cell stimulation (Fig. 4a-f) or hemodynamic changes in response to laser-induced thrombus formation (Fig. $4 \mathrm{~g}-\mathrm{h}$ ).

In a recent study, genetic lineage tracing and intracellular calcium assessment of PDGFRbeta cells over several days unraveled a novel role of renal interstitial cells during proximal tubule regeneration. In that study, the 2-photon laser was used to specifically target and ablate individual proximal tubule cells in the healthy kidney. Laser-induced tubule cell ablation stimulated the immediate activation and migration of adjacent renal interstitial cells toward the site of injury. Furthermore, interstitial cell migration was associated with increased PDGFR-beta cell calcium levels and the local dedifferentiation and proliferation of 
interstitial cell-surrounded proximal tubule cells. Thus, histological staining of the exact same injured proximal tubule segments in fixed tissue (ex vivo histology) confirmed the de novo expression of the dedifferentiation marker CD44 in tubule cells upon interstitial cell recruitment. Consistent with this, proximal tubular regeneration was associated with interstitial cell withdrawal, the loss of epithelial CD44 expression, and functional recovery of the injured tubular segment as determined by in vivo assessment of proximal tubular albumin reabsorption capacity [6]. Hence, a previously unknown, supportive role of renal interstitial cells for tubular regeneration was established by a unique combination of serial intravital MPM over several days, followed by histological analysis of the same renal cells ex vivo.

In the present work, we also provided a detailed step-by-step protocol to accomplish serial intravital MPM of renal cells for several weeks, followed by ex vivo histological analysis to detect cellular biomarkers of the same renal cells in the fixed tissue (F.1-19, Fig. 1f-k). For example, we show how this approach can be used to track the migration of individual cells of the renin lineage (Fig. 3) in homozygous Ren1d-Confetti reporter mice. In this model, cells of the renin lineage co-express two of the four possible Confetti fluorescent proteins (membranous CFP, nuclear GFP, and cytosolic YFP and RFP), which provide a unique color ID of individual cells with one out of ten possible color combinations. Hence, consecutive images of the same glomeruli before and during treatment with a combination of orally given sodium-deficient diet and enalapril clearly showed the migration of cells of the renin lineage, originally located within the parietal epithelial cell layer, into the proximal tubule (Fig. 3). These results are consistent with our recent findings that cells of the renin lineage are migratory [7] and with the recently suggested existence of scattered cells of the renin lineage in the proximal tubule epithelium [34]. Furthermore, ex vivo histology of the same glomeruli including the glomerulartubular junction confirmed the expression of the proximal tubule marker villin in scattered cells of the renin lineage, which had migrated into the proximal tubule (Fig. 3c, f, j).

\subsection{Summary}

Serial intravital microscopy is a powerful tool to track renal cells with high spatial and temporal resolution in vivo. Implantation of a coverslipped titanium ring into the abdominal wall of a mouse, to which the kidney is glued to, provides a temporal optical window through which the same cortical kidney regions can be consecutively imaged noninvasively over several weeks. This allows the tracking of individual renal cells over time and to detect structural changes, such as their migration and proliferation, as well as the associated functional changes. Based on the implantation of an abdominal imaging window, a novel technique was recently established, which further allowed the ex vivo investigation of the same cells for the expression of specific cellular biomarkers. Thus, advanced serial intravital MPM followed by ex vivo histology provides a unique technical approach to track structural, functional, and transcriptional changes of single renal cells during physiological and pathophysiological conditions. Here we provided a detailed step-by-step protocol to accomplish this procedure for further unraveling of mechanisms of renal tissue remodeling. 


\section{Notes}

1. Regarding water tightness of the AIW, the results are more consistent if specialized glass glue is used.

2. The use of optional PLL-g-PEG solution for coating is highly recommended when studying renal immune cell trafficking and homing.

3. The purse-string suture should be applied close to the edges of the incision to ensure that the purse-string-sutured skin and muscle layer fit the groove of the AIW.

4. Test for suitable positioning of the AIW on the kidney surface, before applying glue. Ideal positioning of the AIW allows for maximizing the kidney area that is available for imaging, while limiting pressure of the organ.

5. Continuous blood pressure monitoring is essential when measuring functional renal parameters, such as single-nephron GFR.

6. It is important to place the landmarks in sufficient distance from the regions of interest, in order to avoid damage of the observed nephrons. If glomeruli are studied, it is reasonable to generate laser marks on nearby glomeruli, to ensure that nephrons of interest remain unharmed.

7. To determine the depth of imaging, it is practical to measure the distance of the regions of interest from the kidney capsule, which generates a strong SHG signal.

8. Avoid phototoxicity-induced effects. Perform time-control recordings by applying the same settings without prior laser-induced stimulation to exclude any effects induced by phototoxicity. If photo toxicity was detected, decrease the tissue laser exposure by decreasing laser power and/or the pixel-dwell and total recording time.

\section{References}

1. Peti-Peterdi J, Burford JL, Hackl MJ (2012) The first decade of using multiphoton microscopy for high-power kidney imaging. Am J Physiol Renal Physiol 302(2):F227-F233. 10.1152/ ajprenal.00561.2011 [PubMed: 22031850]

2. Schiessl IM, Castrop H (2016) Deep insights: intravital imaging with two-photon microscopy. Pflugers Arch 468(9):1505-1516. 10.1007/s00424-016-1832-7 [PubMed: 27352273]

3. Diaspro A, Bianchini P, Vicidomini G, Faretta M, Ramoino P, Usai C (2006) Multiphoton excitation microscopy. Biomed Eng Online 5:36 10.1186/1475-925X-5-36 [PubMed: 16756664]

4. Kaissling B, Lehir M, Kriz W (2013) Renal epithelial injury and fibrosis. Biochim Biophys Acta 1832(7):931-939. 10.1016/j.bbadis.2013.02.010 [PubMed: 23466594]

5. Hackl MJ, Burford JL, Villanueva K, Lam L, Susztak K, Schermer B, Benzing T, Peti-Peterdi J (2013) Tracking the fate of glomerular epithelial cells in vivo using serial multiphoton imaging in new mouse models with fluorescent lineage tags. Nat Med 19 (12):1661-1666. 10.1038/nm.3405 [PubMed: 24270544]

6. Schiessl IM, Grill A, Fremter K, Steppan D, Hellmuth MK, Castrop H (2018) Renal interstitial platelet-derived growth factor receptor-beta cells support proximal tubular regeneration. J Am Soc Nephrol 29(5):1383-1396. 10.1681/ASN.2017101069 [PubMed: 29444905] 
7. Kaverina NV, Kadoya H, Eng DG, Rusiniak ME, Sequeira-Lopez ML, Gomez RA, Pippin JW, Gross KW, Peti-Peterdi J, Shankland SJ (2017) Tracking the stochastic fate of cells of the renin lineage after podocyte depletion using multicolor reporters and intravital imaging. PLoS One 12(3):e0173891 10.1371/journal.pone.0173891 [PubMed: 28329012]

8. Ritsma L, Steller EJ, Ellenbroek SI, Kranenburg O, Borel Rinkes IH, van Rheenen J (2013) Surgical implantation of an abdominal imaging window for intravital microscopy. Nat Protoc 8(3):583-594. 10.1038/nprot.2013.026 [PubMed: 23429719]

9. Schiessl IM, Hammer A, Kattler V, Gess B, Theilig F, Witzgall R, Castrop H (2016) Intravital imaging reveals angiotensin ii-induced transcytosis of albumin by podocytes. J Am Soc Nephrol 27(3):731-744. 10.1681/ASN.2014111125 [PubMed: 26116357]

10. Schiessl IM, Kattler V, Castrop H (2015) In vivo visualization of the antialbuminuric effects of the angiotensin-converting enzyme inhibitor enalapril. J Pharmacol Exp Ther 353 (2):299-306. 10.1124/jpet.114.222125 [PubMed: 25680709]

11. Schiessl IM, Bardehle S, Castrop H (2013) Superficial nephrons in BALB/c and C57BL/ 6 mice facilitate in vivo multiphoton microscopy of the kidney PLoS One 8(1):e52499 10.1371/ journal.pone.0052499 [PubMed: 23349687]

12. Burford JL, Villanueva K, Lam L, Riquier-Brison A, Hackl MJ, Pippin J, Shankland SJ, PetiPeterdi J (2014) Intravital imaging of podocyte calcium in glomerular injury and disease. J Clin Invest 124(5):2050-2058. 10.1172/JCI71702 [PubMed: 24713653]

13. Butler MJ, Ramnath R, Kadoya H, Desposito D, Eiquier-Brison A, Ferguson JK, Onions KL, Ogier AS, ElHegni H, Coward RJ, Welsh GI, Foster RR, Peti-Peterdi J, Satchell SC (2019) Aldosterone induces albuminuria via matrix metalloproteinase-dependent damage of the endothelial glycocalyx. Kidney Int 95(1):94-107. 10.1016/j.kint.2018.08.024 [PubMed: 30389198]

14. Peti-Peterdi J, Toma I, Sipos A, Vargas SL (2009) Multiphoton imaging of renal regulatory mechanisms. Physiology 24:88-96. 10.1152/physiol.00001.2009 [PubMed: 19364911]

15. Schiessl IM, Hammer A, Riquier-Brison A, Peti-Peterdi J (2016) Just look! Intravital microscopy as the best means to study kidney cell death dynamics. Semin Nephrol 36 (3):220-236. 10.1016/ j.semnephrol.2016.03.009 [PubMed: 27339387]

16. Hall AM, Rhodes GJ, Sandoval RM, Corridon PR, Molitoris BA (2013) In vivo multiphoton imaging of mitochondrial structure and function during acute kidney injury. Kidney Int 83 (1):7283. 10.1038/ki.2012.328 [PubMed: 22992467]

17. Gyarmati G, Kadoya H, Moon JY, Burford JL, Ahmadi N, Gill IS, Hong YK, Der B, Peti-Peterdi J (2018) Advances in renal cell imaging. Semin Nephrol 38(1):52-62. 10.1016/ j.semnephrol.2017.09.004 [PubMed: 29291762]

18. Weigert R, Porat-Shliom N, Amornphimoltham P (2013) Imaging cell biology in live animals: ready for prime time. J Cell Biol 201 (7):969-979. 10.1083/jcb.201212130 [PubMed: 23798727]

19. Skala M, Ramanujam N (2010) Multiphoton redox ratio imaging for metabolic monitoring in vivo. Methods Mol Biol 594:155-162. 10.1007/978-1-60761-411-1_11 [PubMed: 20072916]

20. Sipos A, Toma I, Kang JJ, Rosivall L, Peti-Peterdi J (2007) Advances in renal (patho) physiology using multiphoton microscopy. Kidney Int 72(10):1188-1191. 10.1038/sj.ki.5002461 [PubMed: 17667980]

21. Kang JJ, Toma I, Sipos A, McCulloch F, Peti-Peterdi J (2006) Imaging the reninangiotensin system: an important target of anti-hypertensive therapy. Adv Drug Deliv Rev 58(7):824-833. 10.1016/j.addr.2006.07.006 [PubMed: 16979787]

22. Kang JJ, Toma I, Sipos A, McCulloch F, Peti-Peterdi J (2006) Quantitative imaging of basic functions in renal (patho)physiology. Am J Physiol Renal Physiol 291(2):F495-F502. 10.1152/ ajprenal.00521.2005 [PubMed: 16609147]

23. Schiess1 IM, Castrop H (2013) Angiotensin II AT2 receptor activation attenuates ATI receptorinduced increases in the glomerular filtration of albumin: a multiphoton microscopy study. Am $\mathrm{J}$ Physiol Renal Physiol 305 (8):F1189-F1200. 10.1152/ajprenal.00377.2013 [PubMed: 23946289]

24. Snippert HJ, van der Flier LG, Sato T, van Es JH, van den Born M, Kroon-Veenboer C, Barker N, Klein AM, van Rheenen J, Simons BD, Clevers H (2010) Intestinal crypt homeostasis results from neutral competition between symmetrically dividing Lgr5 stem cells. Cell 143(1):134-144. 10.1016/j.cell.2010.09.016 [PubMed: 20887898] 
25. Degn SE, van der Poel CE, Firl DJ, Ayoglu B, Al Qureshah FA, Bajic G, Mesin L, Reynaud CA, Weill JC, Utz PJ, Victora GD, Carroll MC (2017) Clonal evolution of autoreactive germinal centers. Cell 170(5):913-926.e919. 10.1016/j.cell.2017.07.026 [PubMed: 28841417]

26. von Massenhausen A, Tonnus W, Himmerkus N, Parmentier S, Saleh D, Rodriguez D, Ousingsawat J, Ang RL, Weinberg JM, Sanz AB, Ortiz A, Zierleyn A, Becker JU, Baratte B, Desban N, Bach S, Schiessl IM, Nogusa S, Balachandran S, Anders HJ, Ting AT, Bleich M, Degterev A, Kunzelmann K, Bornstein SR, Green DR, Hugo C, Linkermann A (2018) Phenytoin inhibits necroptosis. Cell Death Dis 9(3):359 10.1038/s41419-018-0394-3 [PubMed: 29500402]

27. Dunn KW, Sutton TA, Sandoval RM (2012) Live-animal imaging of renal function by multiphoton microscopy. Curr Protoc Cytom Chapter 12:Unitl219 10.1002/0471142956.cy1209s62

28. Gee JM, Smith NA, Fernandez FR, Economo MN, Brunert D, Rothermel M, Morris SC, Talbot A, Palumbos S, Ichida JM, Shepherd JD, West PJ, Wachowiak M, Capecchi MR, Wilcox KS, White JA, Tvrdik P (2014) Imaging activity in neurons and glia with a Polr2a-based and cre-dependent GCaMP5G-IRES-tdTomato reporter mouse. Neuron 83 (5):1058-1072. 10.1016/ j.neuron.2014.07.024 [PubMed: 25155958]

29. Salmon AH, Ferguson JK, Burford JL, Gevorgyan H, Nakano D, Harper SJ, Bates DO, Peti-Peterdi J (2012) Loss of the endothelial glycocalyx links albuminuria and vascular dysfunction. J Am Soc Nephrol 23 (8):1339-1350. 10.1681/ASN.2012010017 [PubMed: 22797190]

30. Nakano D, Kobori H, Burford JL, Gevorgyan H, Seidel S, Hitomi H, Nishiyama A, Peti-Peterdi J (2012) Multiphoton imaging of the glomerular permeability of angiotensinogen. J Am Soc Nephrol 23 (11):1847-1856. 10.1681/asn.2012010078 [PubMed: 22997258]

31. Greka A, Mundel P (2012) Calcium regulates podocyte actin dynamics. Semin Nephrol 32 (4):319-326. 10.1016/j.semnephrol.2012.06.003 [PubMed: 22958486]

32. Eigothier C, Auguste P, Welsh GI, Lepreux S, Deminiere C, Mathieson PW, Saleem MA, Ripoche J, Combe C (2012) IQGAP1 interacts with components of the slit diaphragm complex in podocytes and is involved in podocyte migration and permeability in vitro. PLoS One 7(5):e37695 10.1371/journal.pone.0037695 [PubMed: 22662192]

33. Atkinson BT, Jasuja R, Chen VM, Nandivada P, Furie B, Furie BC (2010) Laser-induced endothelial cell activation supports fibrin formation. Blood 116(22):4675-4683. 10.1182/ blood-2010-05-283986 [PubMed: 20675401]

34. Sequeira Lopez ML, Pentz ES, Nomasa T, Smithies O, Gomez RA (2004) Renin cells are precursors for multiple cell types that switch to the renin phenotype when homeostasis is threatened. Dev Cell 6(5):719-728 [PubMed: 15130496] 

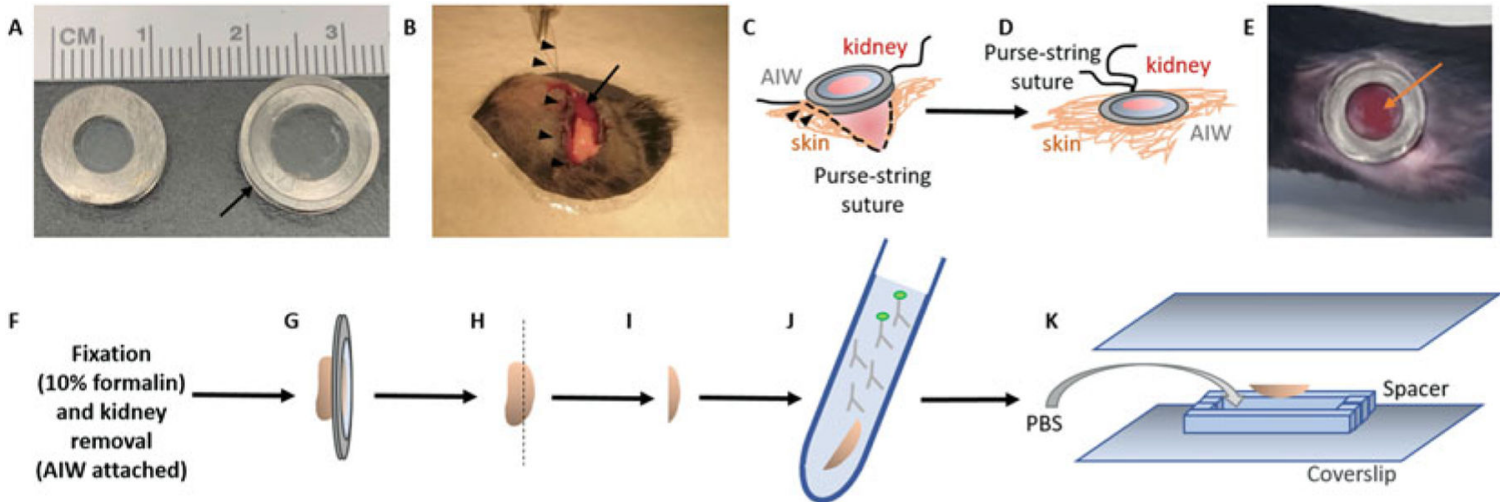

Fig. 1.

Abdominal imaging window (AIW) implantation for intravital imaging of mouse kidney and ex vivo histology. (a) Features of small and large AIW rings. Depending of the body weight of the mouse, $6 \mathrm{~mm}$ inner diameter (for 13-18 g body weight mice) or $8 \mathrm{~mm}$ inner diameter (for $\geq 19 \mathrm{~g}$ body weight mice) AIW rings should be used. The AIWs are made from titanium and consist of an upper and lower ring of $10 \mathrm{~mm}$ (12 $\mathrm{mm}$ for large) outer diameter, which are separated by a $1.5 \mathrm{~mm}$ width groove (arrow). The upper ring of the AIW is covered and sealed by a coverslip using cyanoacrylate glue. (b) Surgical implantation of the AIW. A dorsoventral incision is made above the kidney (arrow), and the abdominal wall is sutured to the skin by a purse-string suture (arrowheads) surrounding the incision. (c, d) Scheme of the surgical implantation of the AIW. The kidney is glued to the coverslip around the edge of the AIW using cyanoacrylate glue. After sufficient waiting time, the sutured abdominal wall and skin is carefully placed into the groove of the AIW, so that its lower ring sinks into the abdomen, while its upper ring is placed on top of the skin (arrowheads, c). (d) The skin is then secured around the groove of the AIW by tightening the purse-string suture. (e) Photograph of the implanted AIW. A large area of the glued kidney surface is attached to the coverslip (arrow). The purse-string suture is fully covered by the upper ring of the AIW, and hence it is sealed and inaccessible. (f-k) Scheme of ex vivo preparation for histology. After perfusion fixation of the tissue (f), the kidney with the attached AIW is carefully removed from the abdominal cavity (g). (h) After gentle removal of the AIW, the kidney area, which was attached to the coverslip, is separated as a 1-2 mm kidney slice (i) and incubated in primary and secondary antibodies for immunohistochemistry (j). For imaging of the fixed and stained tissue in the same region that was imaged in vivo before perfusion, the kidney slice is embedded between two coverslips and a spacer in the correct orientation (k) 

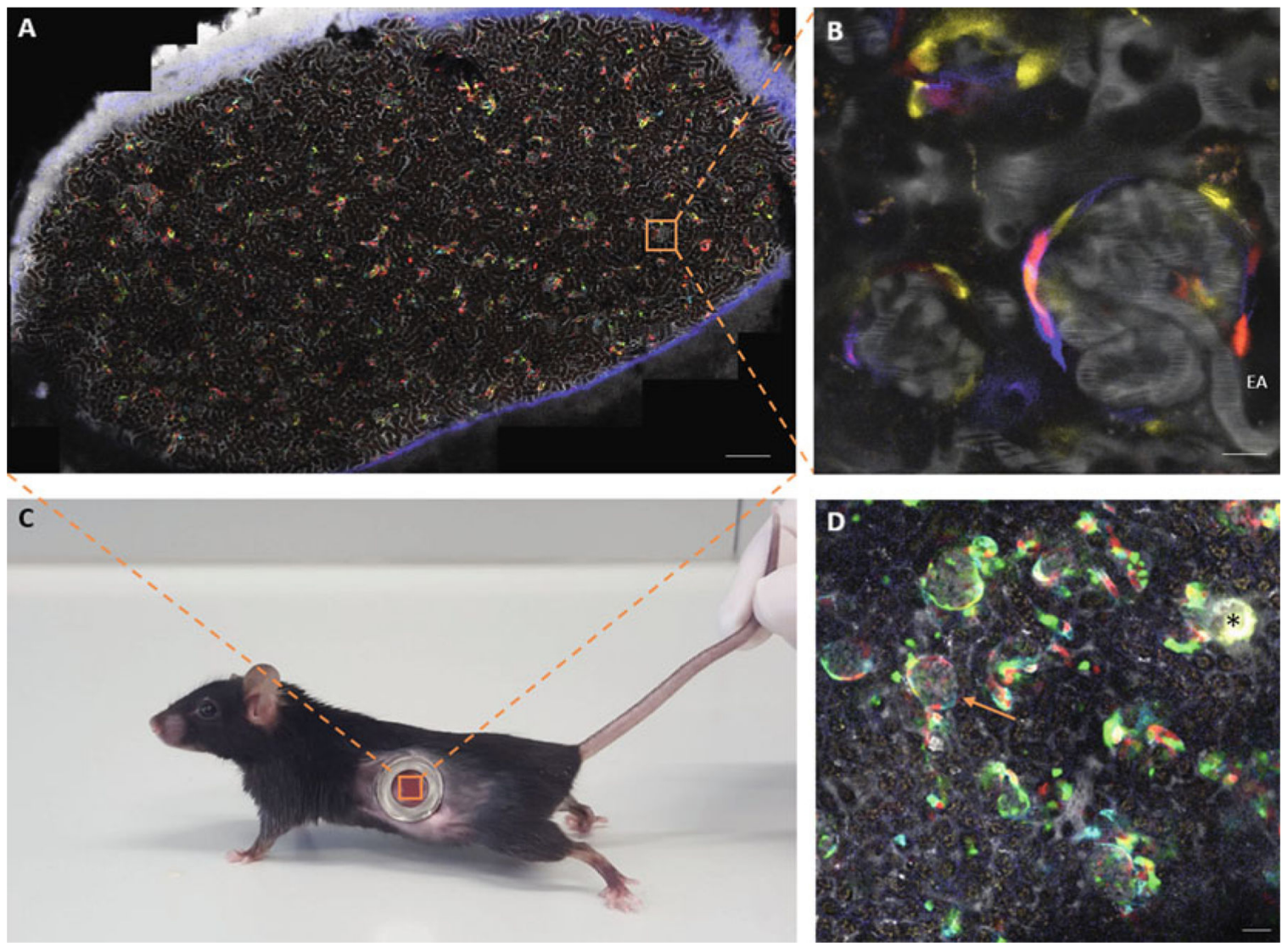

Fig. 2.

Intravital imaging of the mouse kidney using an abdominal imaging window (AIW) and creating a map of the attached kidney surface. (a-c) Overview tile scan (a) and zoom-in area (b) of the cortical kidney tissue attached to the coverslip of an abdominal imaging window, which was implanted into the abdomen of a Ren1d-Confetti mouse (c). The overview tile scan (a) is used as a map to reidentify the same glomerular regions over several weeks. The unique distinguishing pattern of CFP/GFP/YFP/RFP expression in cells of the renin lineage further helps to find the same superficial glomeruli between consecutive imaging sessions. (b) High-resolution image of a Ren1d-Confetti mouse glomerulus, which was identified in the overview scan (a, inset). (d) Example of creating autofluorescent landmarks using laserinduced tissue injury. Tile scan image of a Ren1d-Confetti mouse kidney region shows a laser injury-induced landmark (asterisk) with strong autofluorescence and the glomerulus of interest (arrow) in safe distance from the injury site. To avoid injury of the nephron of interest, a glomerulus of a different nephron was subject to high laser exposure. $E A$ efferent arteriole, $\mathrm{Bar}=100 \mu \mathrm{m}$ in panel $\mathbf{a}$ and $20 \mu \mathrm{m}$ in all other panels 
in vivo imaging Baseline

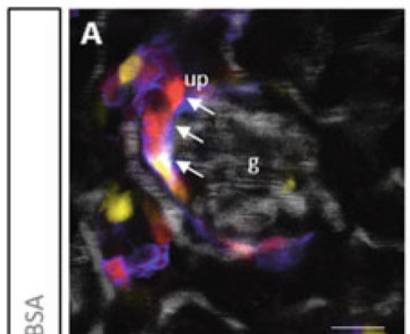

D

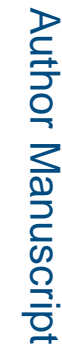

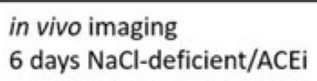
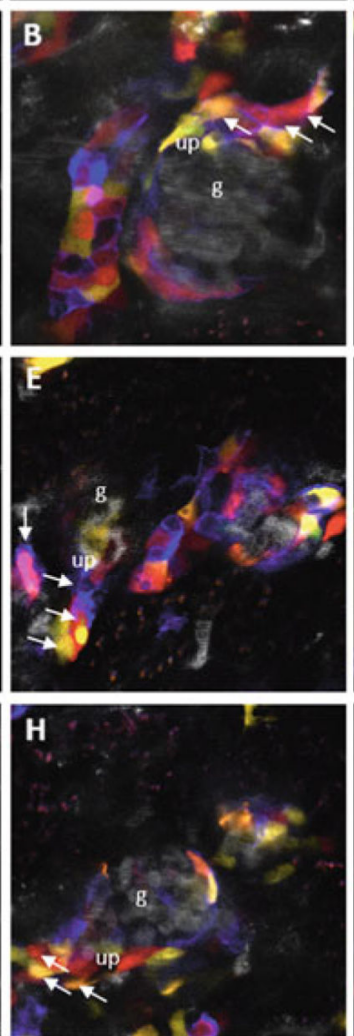

ex vivo histology 6 days $\mathrm{NaCl}$-deficient/ACEi
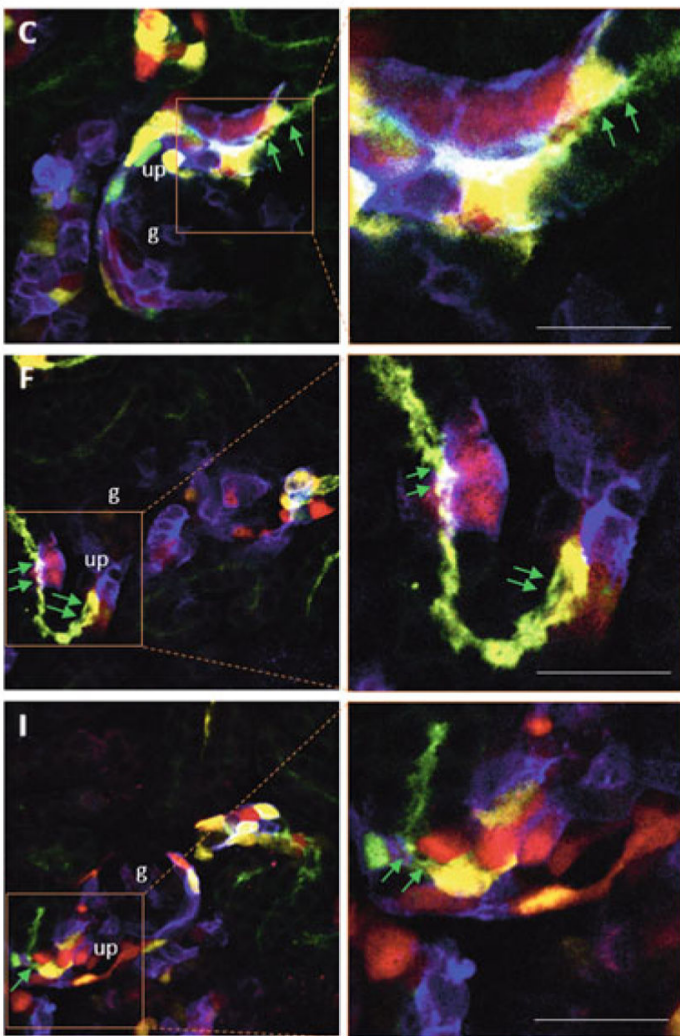

Fig. 3.

Representative images of the combined use of intravital imaging (left columns) and ex vivo histology (right columns) for tracking renal progenitor cell migration and differentiation. Individual renin lineage cells (RLCs) are genetically labeled by the random combination of either cytosolic RFP or YFP, nuclear GFP, or membranous CFP expression in Ren1dConfetti reporter mice. Serial intravital imaging of the same mouse glomerulus (g) and urinary pole (up) before (baseline) and after 6 days of treatment with $\mathrm{NaCl}$-deficient diet and the ACE inhibitor enalapril ( $1 \mathrm{~g} / 1$ via drinking water). The distinct migration of RLCs from the parietal epithelial cell (PEC) layer into the proximal tubule is visible (arrows in $\mathbf{a}-\mathbf{b}, \mathbf{d}-$ $\mathbf{e}$, and $\mathbf{g}-\mathbf{h}$ ). Ex vivo histology allows the reidentification and immunohistological staining of the same cortical kidney regions, which were subject to prior in vivo serial imaging. Endogenous Confetti fluorescence remains preserved. Ex vivo immunostaining for the proximal tubule brush border marker villin (green) confirms the differentiation of some of the recruited RLCs (green arrows in (c, f, i) and magnified insets in right column as shown) into proximal tubule cells. Note that only RLCs in the proximal tubule but not those in glomeruli are billin-positive. Bar $=20 \mu \mathrm{m}$ in each panel 

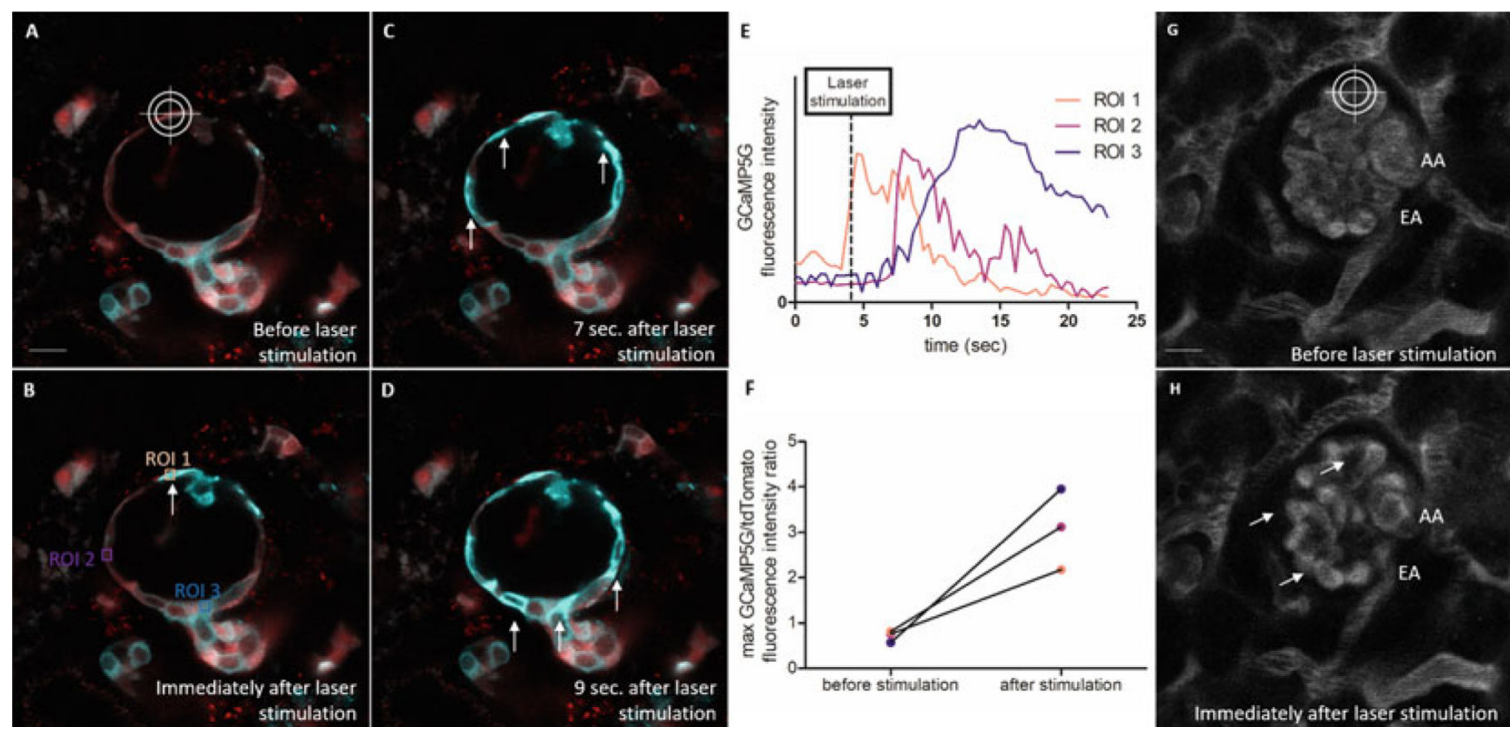

Fig. 4.

Example of using the 2-photon laser as a micromanipulator. (a-d) Intravital MPM images of a Ren1d-GCaMP5 mouse glomerulus before (a) and after laser-induced stimulation (b-d) of a single parietal epithelial cell (PEC, crosshair). Renin lineage cells co-express tdTomato (red) and the fluorescent calcium indicator GCaMP5G (cyan), which indicates real-time elevations of intracellular calcium $\left[\mathrm{Ca}^{2+}\right]$ by increased GCaMP5 fluorescence. Focusing high laser power on a single PEC cell induced a robust and sustained elevation of PEC $\left[\mathrm{Ca}^{2+}\right]$ at the site of injury, which promoted a cell-to-cell propagating $\mathrm{PEC}\left[\mathrm{Ca}^{2+}\right]$ wave along the Bowman's capsule, suggesting that PEC cells are functionally coupled. (e) GCaMP5G fluorescence intensity of regions of interest (ROI) 1-3 (b) over time. (f) Maximal GCaMP5G/tdTomato fluorescence intensity ratios of ROI 1-3 before and after laser-induced PEC cell stimulation. (g, h) Intravital MPM images of a wild-type mouse glomerulus. Plasma is stained after iv injection of Alexa 680-conjugated albumin (gray). Red blood cells are excluded from the staining and appear as dark bands in the lumen of the renal capillaries. Laser-induced stimulation of a glomerular capillary loop induces thrombus formation and severe alterations of glomerular hemodynamics (arrows). $E A$ efferent arteriole, $A A$ afferent arteriole, Bar $=20 \mu \mathrm{m}$ in each panel 


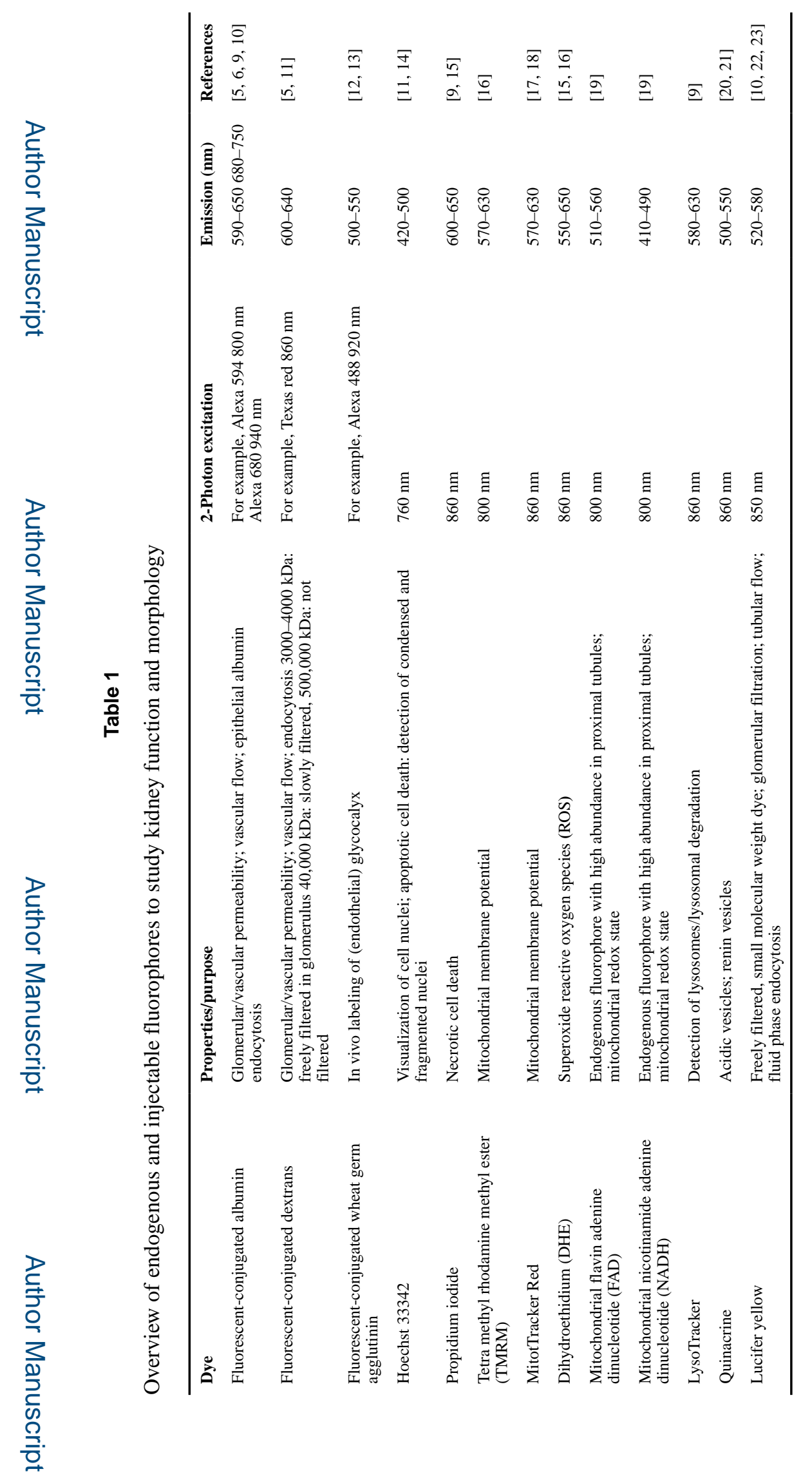

Methods Mol Biol. Author manuscript; available in PMC 2021 January 01. 